\title{
"Genotype-first" approaches on a curious case of idiopathic progressive cognitive decline
}

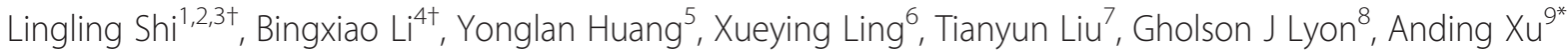
and Kai Wang ${ }^{10,11^{*}}$

\begin{abstract}
Background: In developing countries, many cases with rare neurological diseases remain undiagnosed due to limited diagnostic experience. We encountered a case in China where two siblings both began to develop idiopathic progressive cognitive decline starting from age six, and were suspected to have an undiagnosed neurological disease.
\end{abstract}

Methods: Initial clinical assessments included review of medical history, comprehensive physical examination, genetic testing for metabolic diseases, blood tests and brain imaging. We performed exome sequencing with Agilent SureSelect exon capture and Illumina HiSeq2000 platform, followed by variant annotation and selection of rare, shared mutations that fit a recessive model of inheritance. To assess functional impacts of candidate variants, we performed extensive biochemical tests in blood and urine, and examined their possible roles by protein structure modeling.

Results: Exome sequencing identified NAGLU as the most likely candidate gene with compound heterozygous mutations (chr17:40695717C > T and chr17:40693129A > G in hg19 coordinate), which were documented to be pathogenic. Sanger sequencing confirmed the recessive patterns of inheritance, leading to a genetic diagnosis of Sanfilippo syndrome (mucopolysaccharidosis IIIB). Biochemical tests confirmed the complete loss of activity of alpha-N-acetylglucosaminidase (encoded by NAGLU) in blood, as well as significantly elevated dermatan sulfate and heparan sulfate in urine. Structure modeling revealed the mechanism on how the two variants affect protein structural stability.

Conclusions: Successful diagnosis of a rare genetic disorder with an atypical phenotypic presentation confirmed that such "genotype-first" approaches can particularly succeed in areas of the world with insufficient medical genetics expertise and with cost-prohibitive in-depth phenotyping.

\section{Background}

Next-generation sequencing (NGS) technologies have advanced many aspects of genomic sciences, and have accelerated the pace of discovery in biomedical research [1-3]. For example, NGS has been widely used in population genetics studies [4,5], metagenomics [6,7], agrigenomics $[8,9]$, epigenetics [10] and gene expression studies [11,12]. In particular, human genome and exome sequencing can now be used in dissecting the genetic basis of diseases and

\footnotetext{
* Correspondence: tlil@jnu.edu.cn; kaiwang@usc.edu

${ }^{\dagger}$ Equal contributors

${ }^{9}$ Department of Neurology, The 1st Affiliated Hospital, Jinan University,

Guangzhou, Guangdong 510632, China

${ }^{10}$ Zilkha Neurogenetic Institute, University of Southern California, Los Angeles, CA 90089, USA

Full list of author information is available at the end of the article
}

traits that have proven to be intractable to conventional gene-discovery strategies [13-16]. In addition, NGS empowers clinical diagnostics and other aspects of medical practice, such as genetic diagnosis, disease prognosis, therapeutic target identification, optimization of treatment regimens, prenatal testing and personalized disease-risk profiling.

Besides identifying new mutations in genes previously implicated in known diseases, genome/exome sequencing has also been used to characterize "idiopathic" or "mysterious" diseases, which present phenotypes that may be due to genetic causes. The vast majority of such diseases are indeed "known" diseases that cannot be easily diagnosed by conventional candidate gene approaches, or require complicated differential diagnosis. For example, a recent 
study from the Baylor Whole Genome Laboratory reported a success rate of 25 percent to provide a genetic diagnosis for 250 patients, who presented a range of phenotypes suggesting potential genetic causes and 80\% were children with neurologic phenotypes [17]. An exome sequencing study on 100 patients with intellectual disability found that 53 of them carried de novo mutations, including 13 in known intellectual-disability genes, suggesting that exome sequencing is an effective diagnostic approach for diseases with extensive clinical heterogeneity [18]. Additionally, several studies have reported the discovery of completely novel diseases through genome/exome sequencing. One of the early examples was Ogden syndrome, a previously unreported infantile lethal disorder, involving a mutation in NAA10 identified by us in 2011 using chromosome exon X capture and nextgeneration sequencing [19]. A more recent example is a novel genetic disease which we refer to as "Bookman syndrome", a pediatric onset disease with neuromuscular and cardiac involvement and with clinical features similar to Glycogen Storage Disease Type IV. Although exome sequencing failed to identify the causes for the disease due to technical reasons, we applied genome and transcriptome sequencing and identified a disease-contributory mutation in $R B C K 1$ [20], which was further replicated by another group [21]. Besides finding causal genes for genetic diseases, genome/exome sequencing may also aid in the treatment of rare diseases. One of the earliest highprofile examples is the Pulitzer Prize-winning story of Nicholas Volker, a young boy who at the age of 17 months experienced perineal fistulae coupled with leakage of stool via holes spanning from the intestine to the skin [22]. Exome sequencing on this boy resulted in a diagnosis of an $\mathrm{X}$-linked inhibitor of apoptosis deficiency. Based on this finding, an allogeneic hematopoietic progenitor cell transplant was performed on Volker. Altogether, these examples demonstrated the power of genome/exome sequencing in uncovering the genetic basis of idiopathic diseases and finding treatments for them, even when only a very small number of patient samples are available.

Among people with such diseases, those with idiopathic neuropathy present special challenges for genetic diagnosis $[23,24]$. Due to the heterogeneous nature of clinical presentation, the difficulty with characterizing phenotypes precisely, and the locus heterogeneity for the same diseases, many patients with inherited neuropathy may not obtain a genetic diagnosis by candidate gene testing. However, these patients may be more likely to receive a positive diagnosis by exome sequencing interrogating all genes in a somewhat unbiased manner, and multiple recent studies have demonstrated successful examples $[25,26]$. Frequently, after a candidate gene is identified, it is then found that an atypical phenotype accounts for earlier failed candidate gene approaches, so that genome/exome sequencing can indeed expand the clinical spectrum of previously reported or novel mutations in known disease genes, especially for patients with neuropathy [27]. Perhaps more importantly, genome/exome sequencing can nominate important candidate genes or candidate diseases, which guides the selection of functional assays to confirm the diagnosis of the disease.

In the current study, we describe a case study where exome sequencing together with extensive biochemical tests pinpointed the disease-contributory gene for an idiopathic disease that failed to be diagnosed by conventional means. This study involved a Chinese family quartet (Figure 1) from Guangdong province, a relatively more developed area in China, but even top hospitals in these areas failed to give a diagnosis. It turned out that this is a known disease with a known genetic basis, but with an atypical phenotypic presentation such that appropriate biochemical tests were not performed until mutations pointing to this syndrome were identified. This 'genotypefirst' approach has been discussed before, which suggests the need for the development of large, highly integrated networks of researchers, clinicians, and patient families, with the promise of improved therapies for subsets of patients $[28,29]$. Our study has strong implications for other extremely rare neuropathies, especially in areas where highly experienced medical geneticists may not be readily available.

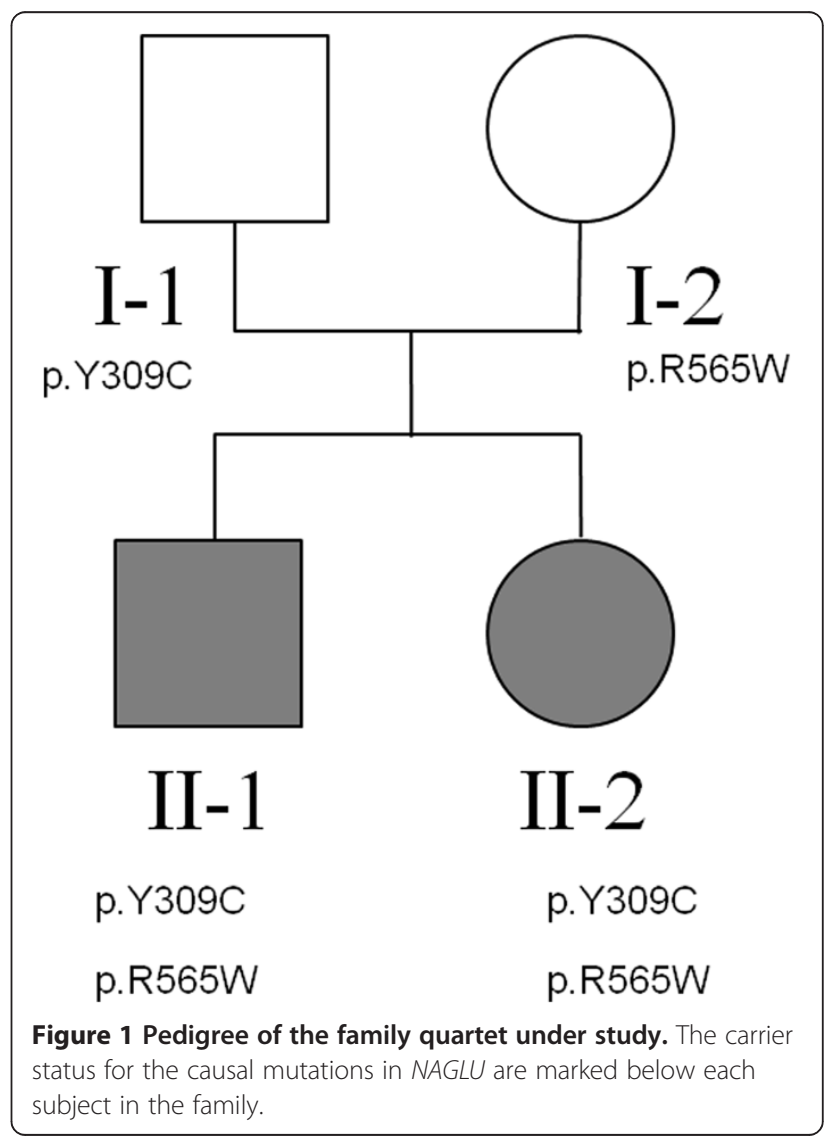




\section{Methods}

\section{Clinical description}

The two probands in our study are siblings from a family in Guangzhou, Guangdong Province in China. The healthy parents are non-consanguineous, and had one affected son and one affected daughter (Figure 1). No evidence of any similar genetic disease was reported in family members. The study to identify disease genes through exome sequencing was approved by Jinan University First Affiliated Hospital Institutional Review Board (reference number: 2013-004). An informed consent for participation of study and publication of genetic results as well as individual medical details was obtained from each family member (or their guardians) who participated in the study.

Individual II- 1 is a 10 year old boy. He was born at term with normal birth parameters and good APGAR scores $(9 / 10 / 10)$. The neonatal period was uneventful, and he had normal motor development during early childhood: he began to look up at 3 months, sit by himself at 5 months, stand up at 11 months, walk at 13 months, and speak at 17 months. He attended a regular kindergarten, without any signs of difference in intelligence, compared to his peers. Starting at age 6 , the parents observed ever increasing behavioral disturbance for the boy, manifesting in multiple aspects of life. For example, he can no longer wear clothes by himself, cannot obey instruction from parents/teachers, can no longer hold subjects tightly in hand, which were all things that he could do before 6 years of age. In addition, he no longer liked to play with others; instead, he just preferred to stay by himself, and he sometimes fell down when he walked on the stairs, which had rarely happened at age 5 . The proband continued to deteriorate: at age 9, he could not say a single word and had no action or response to any instruction given in clinical exams. Additionally, rough facial features were noted with a flat nasal bridge, a synophrys (unibrow), a long and smooth philtrum, thick lips and an enlarged mouth. He also had rib edge eversion, and it was also discovered that he was profoundly deaf and had completely lost the ability to speak. He also had loss of bladder control. The diagnosis of severe intellectual disability was made, based on Wechsler Intelligence Scale examination. Brain MRI demonstrated cortical atrophy with enlargement of the subarachnoid spaces and ventricular dilatation (Figure 2). Brainstem evoked potentials showed moderate abnormalities. Electroencephalography (EEG) showed abnormal sleep EEG.

Individual II-2 is a 9 years old girl. She was born at term, also with normal birth parameters. She began to stand at 11 months, walk with aid at 13 months, and speak at 17 months. At age 5, she was just like other children of similar age, with the ability to dress and sing, and count by herself. Starting at 6 years of age, she began to show regression of developmental patterns: she could not dress by herself anymore, and could not express even a single sentence or count numbers. Clinical examination revealed a coarse face with low anterior and posterior hairlines, prominent frontal bossing, thick eyebrows, synophrys (unibrow), hypertelorism, and thick lips. Growth parameters were normal. Her clinical course was also severe, with progressive neurodegeneration, behavioral problems (including hyperactivity, impulsivity, obstinacy, anxious behaviors and autistic-like behaviors), and hearing loss. The diagnosis of severe intellectual disability was made, based on Wechsler Intelligence Scale examination. Measuring activities of daily living showed extreme disability. Brain MRI demonstrated cortical atrophy with enlargement of the subarachnoid spaces and ventricular dilatation (Figure 2). Brainstem evoked potentials showed moderate abnormalities. EEG recording showed abnormal sleep EEG, just like her brother's manifestation.

\section{Exome sequencing}

We elected to perform exome sequencing on the probands, and to use parental samples for Sanger validation. Genomic DNA was extracted from peripheral blood for all family members by Qiagen DNeasy Blood \& Tissue kit (Valencia, CA, USA). We obtained at least 5ug DNA from each sample, and all DNA samples passed quality control measures for exome sequencing.

Exome capture was carried out on $1 \mu \mathrm{g}$ of genomic DNA, using the Agilent SureSelect ${ }^{\mathrm{XT}}$ Human All Exon $50 \mathrm{Mb}$ kit (Agilent Technologies, Santa Clara, CA, USA), which targets $50 \mathrm{Mb}$ of coding exonic sequences, which were annotated by the GENCODE project, CCDS, miRbaseV14 and Sanger, as well as 10 base pairs of flanking sequence of each exonic region and non-coding RNA. The captured and amplified library was then loaded onto the Illumina Hiseq2000 sequencer. We generated $100 \mathrm{bp}$ paired-end reads in the sequencing run, according to the manufacturer's protocols. Raw images were processed by Illumina Pipeline v1.3.4 for basecalling with default parameters. Illumina SCS and CASAVA software were used for raw data processing and FASTQ file generation. In total, we obtained $\sim 70$ million reads per sample.

\section{Sequence alignment and variant calling}

For bioinformatics analysis of the FASTQ data from exome sequencing, we used SeqMule (http://seqmule.usc. $\mathrm{edu}$ ), which is an automated pipeline to execute multiple alignment algorithms and multiple variant calling software tools. Briefly, sequencing data was evaluated with FastQC (http://www.bioinformatics.babraham.ac.uk/projects/fastqc/). Short reads were aligned to reference genome (hg19) by BWA-MEM (version 0.7.4) [30] algorithm with default settings. Then we used three variant calling software tools, including the GATK version 3.1 

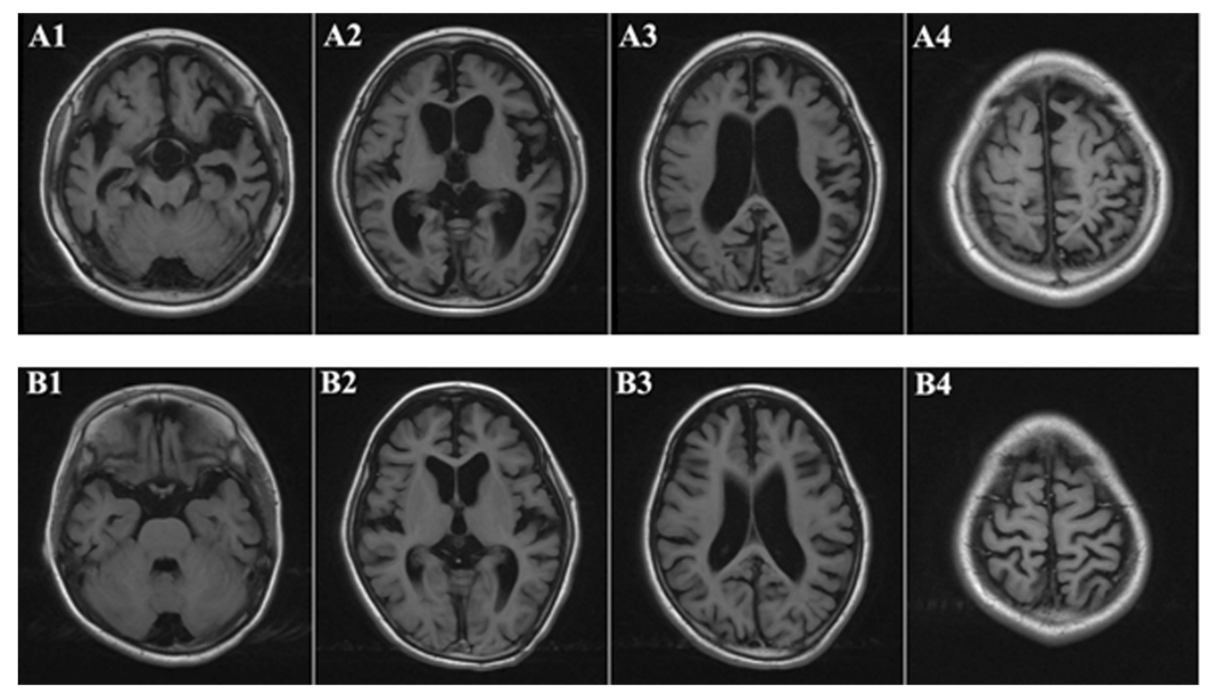

Figure 2 Brain MRI of the two prodbands (A: brother 10 years old; B: sister 9 years old): Axial T1-weighted images showed that the hemisphere sulci and schizencephaly general broadened and deepened. Double lateral and the third ventricle, cisterns were significantly dilated. The gray matter and white matter of the two hemisphere decreased. All of the above changes indicated ventricular dilatation and cortical atrophy in the two probands.

[31], SAMtools version 0.1.19 [32] and FreeBayes version 0.9.14 [33] with default settings for exome sequencing. We selected the consensus variants using the "two of three algorithms" protocol, which specifies that variants that were detected by at least two algorithms will be present in the final consensus calling set.

\section{Variant annotation and prioritization}

We used the ANNOVAR software [34] for functional annotation of variants and the Tute web application (tutegenomics.com) for identifying candidate variants and genes for the clinical phenotype. We used a "singlecase analysis" pipeline to identify a list of candidate genes with the following criteria: (1) identify variants causing splicing or protein coding changes, including stop loss and stop gain variants; (2) remove variants with minor allele frequency (MAF) greater than $1 \%$ in the PopFreqMax database from ANNOVAR package [34], which include maximum allele frequency from the 1000 Genomes Project April 2012 release (five ethnicity groups), the NHLBI-6500 Exomes (two ethnicity groups), and the Complete Genomics 46 genomes (CG46) database; (3) imposed a recessive mode of inheritance, with at least two deleterious mutations found in each candidate genes in the proband, and with the mutations shared by both probands; (4) The resulting variants and genes were sorted based on relevance to user-supplied phenotypes, as well as annotations from clinical databases such as ClinVar (version 20140303) and HGMD (version 2014_2) [35].

\section{Validation by Sanger sequencing}

Selected putative variants were examined among all family members using Sanger sequencing. Given the chromosomal position of variants, we designed PCR primers to amplify fragments harboring individual variants by Primer3 [36]. The PCR primers were designed to encompass the candidate position, ensuring that common SNPs are not covered by the primers. The ABI $3730 \mathrm{XL}$ sequencer was used for sequencing, and the resulting *AB1 files were loaded into the ABI Sequence Scanner Software v1.0 for further analysis and genotype calling. All sequence traces were manually reviewed to ensure the reliability of the genotype calls.

The primers used for chr17:40695717(C > T) mutation are CCCGCCTCTTCCCCAACTC (forward) and GGACGCCTCCAGCCCTCAA (reverse). The primers used for chr17:40693129 (A > G) mutation are AAACC AGGAGCTGTAGAGAAGT (forward) and CTGCCTA CCCCTACTGACATCT (reverse).

\section{Structure modeling}

We searched the Protein Data Bank (PDB) [37] for structure models of NAGLU. Although the structure for human NAGLU was not available, a previous study solved the crystal structure, catalytic mechanism, and inhibition of CpGH89 from Clostridium perfringens, a close bacterial homolog of NAGLU [38]. We downloaded the structure from X-ray diffraction with a resolution of $2.36 \AA$ with binding of the ligand 2-Acetamindo-1,2-Dideoxynojirmycin (PDB identifier: 2VC9). We built a structure model for 
human NAGLU using I-TASSER [39], given high sequence identity. The template structure 2VC9 binds to 2-ACETAMIDO-1,2-DIDEOXYNOJIRMYCIN (PDB identifier: NOK). The position of NOK is decided by superpositioning our model to the template structure 2VC9 (shown in ball and stick). The 3D representation of the structure was generated by PYMOL (http://www.pymol.org/).

\section{Biochemical test in blood/urine}

Enzyme activity was measured on peripheral blood leukocytes using fluorogenic substrate obtained from Moscerdam Substrates (Rotterdam, Netherlands); peripheral blood leukocytes was collected and sonicated to protein homogenates. Protein homogenates were incubated with Moscerdam substrate. The parameters for enzyme activity was obtained by measuring the fluorescence intensity and comparing with standard fluorescence intensity and concentration. The assays were performed according to the protocol at Department of Endocrinology and Metabolism, Guangzhou Women and Children's Medical Center [40-44]. Urinary GAG was measured using dimethylmethylene blue/Tris by spectrophotometry and corrected for urinary creatinine $(\mathrm{Cr})$ content $[45,46]$. The ratio of GAG/Cr ( $\mathrm{mg} / \mathrm{mmol})$ was compared to age-matched normal controls $(6 \mathrm{~m}-12 \mathrm{~m},<23.3 ; 12 \mathrm{~m}-36 \mathrm{~m},<17.3 ; 3-5$ years, $<13.2$; $>5$ years, $<9.7)$.

\section{Results}

\section{Assessment of clinical information}

The two probands were brother and sister one year apart in age, and both of them had perfectly normal development patterns in the first five years of age. Starting from age six, the probands suddenly began to develop progressive speech and cognitive decline with behavioral difficulties, motor function decline and hearing loss: they gradually lost the ability to talk, count, or walk. Rough facial features began to develop including synophrys (unibrow), thick lips and enlarged mouth. Brain MRI demonstrated cortical atrophy with enlargement of the subarachnoid spaces and ventricular dilatation (Figure 2). An extended list of clinical features is given in Materials and Methods. In the course of the clinical workup, multiple attempts for genetic diagnosis were made, including tests of chromosomal abnormalities and sequencing on a panel for metabolic diseases. Multiple types of blood screening tests and physical examinations were performed on the pedigree, especially the probands, to exclude the common inherited metabolic diseases and nervous system abnormalities. Additional factors were considered to exclude the possibility of food poisoning, environmental toxification and other non-genetic reasons. However, none of these attempts showed positive results, and these two probands were eventually referred to as having "mental deterioration of unknown origin".
To research the causes of the diseases in this family, we examined all available phenotype information on the probands. Both parents are healthy and are nonconsanguineous. No evidence of other genetic disease was reported in any additional family members. We suspected that this is an extremely rare or novel genetic disease, possibly following a recessive pattern of inheritance. Exome sequencing appeared to be the next natural choice to identify candidate mutations possibly contributing to this idiopathic disease.

\section{Exome sequencing identifies a prioritized list of candidate mutations}

To identify genetic causes for the disease in the family, we performed exome sequencing on the two probands with Agilent exon capture arrays, followed by sequencing on the Illumina HiSeq2000 platform. For each proband, we obtained approximately 70 million paired-end reads of 100 base pairs. The mapping rates for reads from brother (II-1) and sister (II-2) were 98.4\% and $98.8 \%$, respectively, and the on-target rates (fraction of reads mapped to designed exome regions) were $54.9 \%$ and $58.4 \%$, respectively. We achieved a coverage of $68.2 \mathrm{X}$ and $71.3 \mathrm{X}$ over designed capture regions, and with $88.3 \%$ and $88.5 \%$ of target regions covered by $\geq 10$ reads for brother and sister, respectively $(82.0 \%$ and $82.8 \%$ and covered by $\geq 20$ reads). Data analysis was performed using the SeqMule pipeline (http://seqmule.usc. edu), which is an automated pipeline for analysis of high-throughput sequencing data. It integrates multiple alignment algorithms and multiple variant calling software tools and gives user the flexibility to choose their preferred aligners and variant callers, and enables generation of consensus calls from multiple calling algorithms to improve reliability. We selected the consensus variants using the "two of three algorithms" protocol from GATK v3.1 [31], SAMtools v0.1.19 [32] and FreeBayes v0.9.14 [33], based on our previous work showing improved accuracy when using multiple calling algorithms [47]. The overlap of the three calling algorithms were presented in Figure 3, demonstrating substantial differences between the variant callers, even when the same alignment files were used. In total, 37,183 (2,268 indels) and 37,822 (2,312 indels) variants were identified from brother and sister respectively. To further identify likely causal genetic variants from exome data, we used the Tute web server which incorporates phenotype information, a variant scoring system and several clinical databases to rank variants and genes. NAGLU stands out as the most likely candidate gene for the disease under a recessive model (by requiring two predicted deleterious mutations in the same gene).

We identified two compound heterozygous mutations in NAGLU (N-acetylglucosaminidase, alpha), in both the 


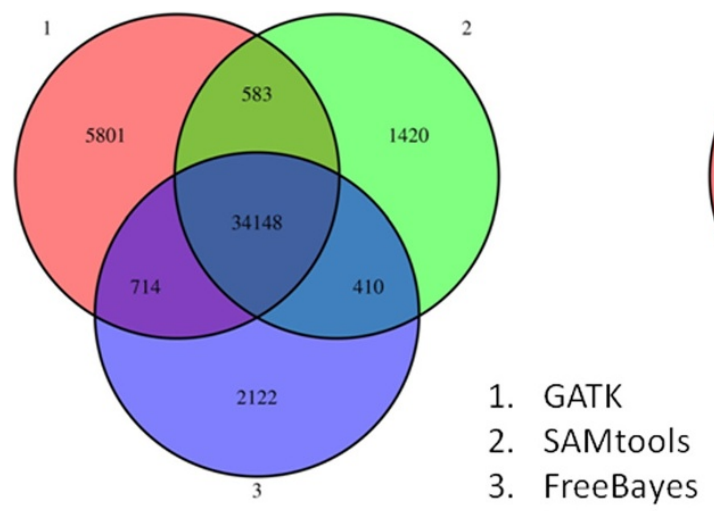

II-1

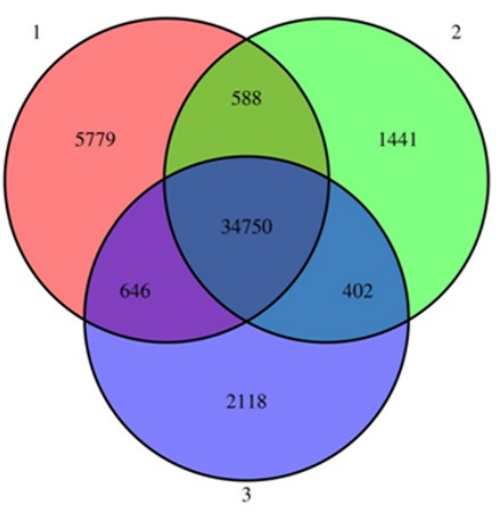

II-2

Figure 3 Venn diagrams showing the overlap of different variant calling algorithms on two exomes (II-1 and II-2). The three algorithms are (1) GATK, (2) SAMtools and (3) FreeBayes. The numbers denote the number of called variants.

probands (chr17:40695717C > T, c.1693C > T, p.R565W; chr17:40693129A > G, c.926A > G, p.Y309C). Neither variant was reported in the 1000 Genomes Project, the NHLBI-ESP6500 exome sequencing project or the Complete Genomics 46 genomes database. The first SNP was documented in dbSNP with identifier rs104894597. We evaluated the bioinformatics predictions on deleteriousness of non-synonymous variants from the dbNSFP database [48], and found that all algorithms gave largely consistent predictions that both are deleterious (Table 1), except that LRT predicted p.Y309C as "neutral". NAGLU encodes a lysosomal enzyme that degrades heparan sulfate by hydrolysis of terminal $\mathrm{N}$-acetyl-D-glucosamine residues in $\mathrm{N}$-acetyl-alpha-D-glucosaminides, which is the fifth step of degradation of glycosaminoglycans (mucopolysaccharides). Defects in this gene are the cause of mucopolysaccharidosis type IIIB (MPS-IIIB), also known as Sanfilippo syndrome B [49]. This disease is characterized by the lysosomal accumulation and urinary excretion of heparan sulfate. The clinical severity of MPS-IIIB is highly variable, ranging from mild to severe, even in the same family, but typically includes progressive neurodegeneration, behavioral problems, mild skeletal changes, and shortened life span [50-52]. To date, over 119 unique disease contributory mutations underlying MPS IIIB have been identified in NAGLU in the Human Gene Mutation Database (HGMD) [53]. Both mutations in the probands were documented in HGMD as previously reported diseasecontributory mutations $[54,55]$. Only one mutation (p. R565W) was documented in the ClinVar database (identifier: RCV000001633) as a "pathogenic" mutation.

\section{Sanger validation of the candidate variants}

To validate the presence of the mutations and to identify whether they are recessively inherited, we performed Sanger sequencing on all the family members
(Figure 4A,B). We found that the father and mother carried the p.Y309C and p.R565W and mutations, respectively, while the two probands inherited both variants from their parents, resulting in compound heterozygous mutations in NAGLU. Therefore, Sanger validation confirmed that these two mutations are inherited from different parents, which fits the known inheritance mode of the Sanfilippo syndrome.

Table 1 Bioinformatics predictions of deleteriousness on the two non-synonymous variants in NAGLU

\begin{tabular}{|c|c|c|}
\hline & p.Y309C & p.R565W \\
\hline SIFT score & 0 & 0 \\
\hline SIFT prediction & Deleterious & Deleterious \\
\hline PolyPhen score & 1 & 1 \\
\hline PolyPhen prediction & Probably damaging & Probably damaging \\
\hline LRT score & 0 & 0 \\
\hline LRT prediction & Neutral & Deleterious \\
\hline Mutation Taster score & 1 & 1 \\
\hline Mutation Taster prediction & Disease causing & $\begin{array}{l}\text { Disease causing } \\
\text { automatic }\end{array}$ \\
\hline Mutation Assessor score & 3.55 & 3.46 \\
\hline Mutation Assessor prediction & High & Medium \\
\hline FATHMM score & -6.27 & -5.82 \\
\hline FATHMM prediction & Deleterious & Deleterious \\
\hline MetaSVM score & 1.05 & 1.05 \\
\hline MetaSVM prediction & Deleterious & Deleterious \\
\hline MetaLR score & 0.99 & 0.98 \\
\hline MetaLR prediction & Deleterious & Deleterious \\
\hline Tute score & 0.92 & 0.92 \\
\hline Tute prediction & Deleterious & Deleterious \\
\hline
\end{tabular}



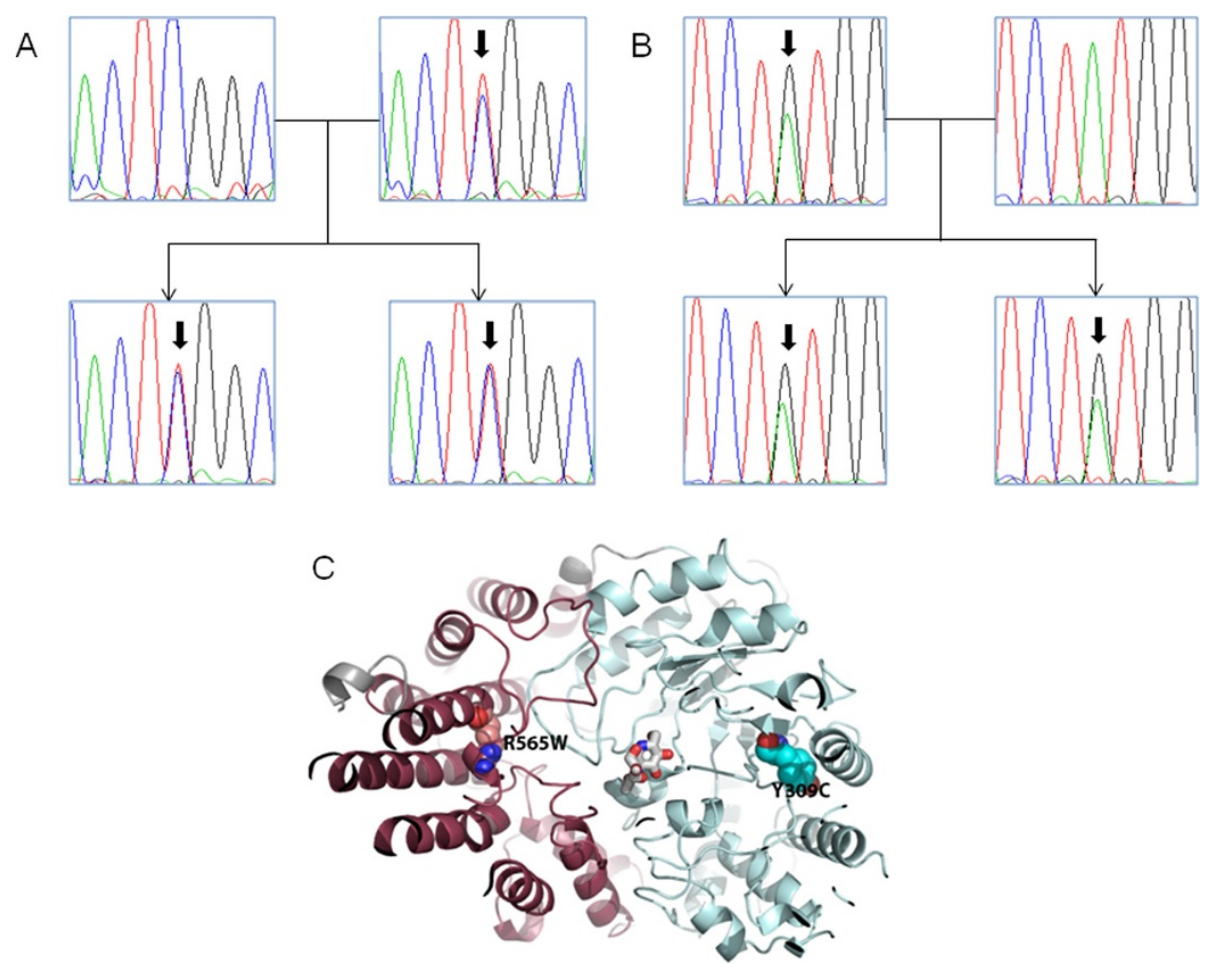

Figure 4 Sanger validation and structural modeling of the two compound heterozygous candidate variants in NAGLU in the family quartet. (A) Validation of the chr17:40695717(C > T) mutation by Sanger sequencing in the family. (B) Validation of the chr17:40693129 (A > G) mutation by Sanger sequencing in the family. (C) Structural location of mutation R565W and Y309C in NAGLU. We have built a structure model based on a close homolog structure (PDB identifier: 2VC9) of CpGH89 from Clostridium perfringens. The template structure 2VC9 binds to 2-ACETAMIDO-1,2-DIDEOXYNOJIRMYCIN (PDB identifier: NOK).

\section{Functional impacts of the candidate variants by biochemical test}

The analysis of candidate genes guided us to perform functional studies to evaluate whether MPS-IIIB or other types of MPS could explain the observed disease phenotypes in the family. We tested eight MPS related enzymes, including alpha-L-iduronidase, iduronate-2-sulfatase, heparan sulfate sulfatase, alpha $\mathrm{N}$-acetylglucosaminidase, galactosamine 6-Sulfatase, $\beta$-galactosidase, arylsulfatase, $\beta$ glucuronidase, which are responsible for different subtypes of MPS (Table 2). The biochemical tests showed that alpha-N-acetylglucosaminidase, which is encoded by $N A G L U$, is completely inactive in both probands. Additionally, the level of alpha-L-iduronidase was slightly lower than reference value in one proband, but this could be due to variations in measurement. The biochemistry results were consistent with the bioinformatics analysis, and demonstrated that the two compound heterozygous mutations are indeed loss-of-function mutations.

To further validate the consequences of the lack of enzyme activity for alpha- $\mathrm{N}$-acetylglucosaminidase, we performed urinary tests to measure the levels of metabolites for the enzyme (Table 3). The level of urinary glucosaminoglycan was extremely high in both probands, as opposed to an expected value of zero. Additionally, the substrates of the NAGLU enzyme, including dermatan sulfate and heparan sulfate, are both positive in urine, further suggesting that loss of function mutations in NAGLU leads to abnormal accumulation of sulfate in urine, which may explain the observed neuropathy.

\section{Functional impacts of the candidate variants by structural modeling}

To further understand the functional impacts of the two variants, we attempted to examine structure models for NAGLU. A previous study solved the crystal structure, catalytic mechanism, and inhibition of CpGH89 from Clostridium perfringens, a close bacterial homolog of NAGLU [38]. The structure was generated by X-ray diffraction with a resolution of $2.36 \AA$. We built a structure model for human NAGLU based on this template (PDB identifier: 2VC9) using I-TASSER [39], given the high sequence identity. The complete structure contains four domains (Figure 4C), including a N-terminal domain which is a putative family 32 carbohydrate-binding module (CBM) with the typical $\beta$-sandwich fold, a catalytic region comprises a small $\alpha / \beta$ domain, an elaborated $(\alpha / \beta) 8$ barrel, and an all $\alpha$-helical domain that packs against the first 
Table 2 Results of biochemical tests on the activity of MPS-related enzymes

\begin{tabular}{|c|c|c|c|c|c|c|c|}
\hline Disease subtype & Enzyme & Gene & Value (sister) & Value (brother) & Reference value & Result & Unit* \\
\hline MPSI & alpha-L-iduronidase & IDUA & 26.5 & 25.1 & $25.4-118.5$ & Borderline abnormal & $\mathrm{nmol} / \mathrm{mg} / \mathrm{h}$ \\
\hline MPSII & iduronate-2-sulfatase & IDS & 31.4 & 32.2 & $30-120$ & normal & $\mathrm{nmol} / \mathrm{mg} / 4 \mathrm{~h}$ \\
\hline MPSIIIA & heparan sulfate sulfatase & SGSH & 5.3 & 6.3 & $4.1-12$ & normal & $\mathrm{nmol} / \mathrm{mg} / 17 \mathrm{~h}$ \\
\hline MPSIIIB & $\begin{array}{l}\text { Alpha-N-acetyl- } \\
\text { glucosaminidase }\end{array}$ & NAGLU & 0.0 & 0.0 & $5-22$ & abnormal & $\mathrm{nmol} / \mathrm{mg} / 17 \mathrm{~h}$ \\
\hline MPSIVA & $\begin{array}{l}\text { galactosamine } \\
\text { 6- sulfate Sulfatase }\end{array}$ & GAS & 72.2 & 68.1 & $40-170$ & normal & $\mathrm{nmol} / \mathrm{mg} / 17 \mathrm{~h}$ \\
\hline MPSIVB & $\beta$-galactosidase & $\operatorname{lac} Z$ & 89.2 & 88.5 & $50.3-140.7$ & normal & $\mathrm{nmol} / \mathrm{mg} / \mathrm{h}$ \\
\hline MPSVI & arylsulfatase & Ars & 67.0 & 49.0 & $50.4-175.2$ & normal & $\mathrm{nmol} / \mathrm{mg} / \mathrm{h}$ \\
\hline MPSVII & $\beta$-glucuronidase & GUSB & 94.0 & 98.0 & $38.1-202.5$ & normal & $\mathrm{nmol} / \mathrm{mg} / \mathrm{h}$ \\
\hline
\end{tabular}

${ }^{*}$ concentrations of metabolites $(\mathrm{nmol} / \mathrm{mg})$ after hour of incubation.

three domains. We found that these two mutations were not directly within the active site (ligand-binding site) of NAGLU. Mutation Y309C is in the middle of the elaborated alpha/beta barrel (in blue) and R565W is located at the alpha-helical domain (in red) that packs against the binding site. Therefore, mutation Y309C may affect the structural stability of the alpha/beta barrel that constitutes the bottom of the active site. Mutation R565W may plays an important role in structural packing of the alphahelical domain that contributes to the stability of the complete structure.

\section{Discussion}

In this study, we present a case illustrating the power of NGS in clinical diagnosis of a rare disease in two siblings with somewhat atypical phenotypes, with a disease onset at $\sim$ six years of age. Although this turned out to be a known disease with a known genetic cause, our experience in this study is nevertheless revealing in the context of a study conducted in China, where traditionally experienced medical geneticists are lacking. We suspected that had a more experienced medical geneticist reviewed the patient, biochemical tests on different types of MPS could have been ordered, and a diagnosis of MPS-IIIB may have been made without exome sequencing. However, in practice, numerous other tests were ordered and all turned out to be negative, to a point where the family was reluctant to pay for any additional tests. After exome sequencing, the mutation pointed us to a phenotype related to MPS, and therefore we performed biochemical tests in blood and urine to confirm this diagnosis. Therefore, exome sequencing in this particular context provided guidance for us to reach a final diagnosis, and this also illustrates the importance of supporting bioinformatics predictions with functional evidence.

Sanfilippo syndrome B (or MPS-IIIB) is an extremely rare disorder and the prevalence in China is unreported and unknown. Due to the rare nature of the disease, the incidence rate could vary significantly among different parts of the world. Using multiple ascertainment sources, one study obtained an incidence rate for Sanfilippo syndrome (form $\mathrm{A}+\mathrm{B}+\mathrm{C}$ ) in western Australia for the period 1969 to 1996 of approximately 1 in 58,000 live births [56]. Since there were 5 people with type B in the total of 11 cases, this suggested an incidence of MPS-IIIB of approximately 1 in 127,600 live births. In different studies, an incidence of 1:24,000 was reported for all subtypes of MPS III [52]. A more recent study on the Australian population estimated an incidence rate of 1 in 200,000 [57]. Northern Ireland tends to have much lower incidence of MPS, and a report showed the rate of 1 in 280,000 for all subtypes of MPS III [58]. In the Netherland, the combined birth prevalence for all subtypes of MPS-III is 1.89 per 100,000 live births [59].

While we emphasize that the development of technologies has now enabled rapid and relatively cost-

Table 3 Results from urinary test of glycosaminoglycan related parameters

\begin{tabular}{|c|c|c|c|c|c|}
\hline Urinary test & Value (sister) & Value (brother) & Reference value & Result & Unit \\
\hline Urinary glycosaminoglycan (GAG) & 528.1 & 413.8 & 0 & abnormal & $\mathrm{mg} / \mathrm{L}$ \\
\hline Urinary glycosaminoglycan/Creatinine ( $\mathrm{GAG} / \mathrm{Cr}$ ) & 36.7 & 39.7 & $<8.4$ & abnormal & $\mathrm{mg} / \mathrm{mmol}$ \\
\hline Dermatan sulfate & + & + & - & abnormal & \\
\hline Heparan sulfate & + & + & - & abnormal & \\
\hline Keratan & - & - & - & normal & \\
\hline Chondroitin sulfate & - & - & - & normal & \\
\hline
\end{tabular}


efficient identification of genetic causes in all parts of the world, the major bottleneck is still the identification of disease-contributory variants, which is heavily dependent on the bioinformatics analysis of raw data and functional interpretation of genetic variants. We used the SeqMule pipeline for variant calling, to take ' 2 out of 3 ' consensus calls from three variant calling algorithms, in a way similar to what the 1000 Genomes Project has been implementing in their variant calling procedure. We also used ANNOVAR and the Tute web server for variant analysis focusing on missense, nonsense and splice variants. For the two missense variants in $N A G L U$, we used multiple prediction algorithms, including six from dbNSFP [48] (SIFT, PolyPhen, LRT, Mutation Taster, Mutation Assessor, FATHMM), two meta-scores built by us (MetaSVM, MetaLR), and the Tute score (tutegenomics.com), and found that these algorithms gave consistent predictions. However, the main reason we focused on NAGLU is that it is reported in clinical databases (ClinVar and HGMD) as containing mutations linked to disease. This fact underscores the importance of curated mutation databases in helping identify disease causal mutations from newly sequenced genomes or exomes.

\section{Conclusions}

In conclusion, we have presented an example in China where exome sequencing preceded and guided the selection of functional validations, to lead to a diagnosis of a known disease with a somewhat atypical presentation. With the ever decreasing cost of NGS and the rapid adoption of NGS in clinical settings, we expect that genome/exome sequencing will be increasingly useful in the diagnosis of rare genetic disorders with atypical phenotypic presentations, including in areas of the world where experienced medical geneticists are scarce.

\section{Competing interests}

K.W. is board member and share holder of Tute Genomics, Inc. G.J.L serves on the medical advisory board of GenePeeks, Inc.

\section{Authors' contributions}

LS performed experiments and carried out data analysis. BL characterized samples and contributed materials. YH performed biochemical tests. XL performed brain MRI analysis. TL performed structural modeling experiments. GJL advised on phenotype analysis and data analysis. AX and KW conceived the study and guided data interpretation. LS and KW wrote the manuscript. All authors read and approved the final manuscript.

\section{Acknowledgements}

The authors thank the probands and their family members for donating blood samples for scientific research, and for providing detailed phenotype information and medical records. We thank Dr. Feng Wang (Wuhan Institute of Technology and Kaigene Technology) for help with data analysis and archival. L.S. is supported by the Fundamental Research Funds for the Central Universities of China (21614331), the Medical Science Foundation of Guangdong Province (B2014213) and the National Natural Science Foundation of China (31400922). K.W. is supported by NIH/NHGRI grant number R01 HG006465. G.J.L is supported by funds from the Stanley Institute for Cognitive Genomics at Cold Spring Harbor Laboratory.

\section{Author details}

'Guangdong-Hongkong-Macau Institute of CNS Regeneration, Jinan University, Guangzhou, Guangdong 510623, China. ${ }^{2}$ Guangdong Medical Key Laboratory of Brain Function and Diseases, Jinan University, Guangzhou, Guangdong 510623, China. ${ }^{3}$ GHM Collaboration and Innovation Center for Tissue Regeneration and Repair, Jinan University, Guangzhou, Guangdong 510623, China. ${ }^{4}$ Neonatal Intensive Care Unit, The 1st Affiliated Hospital, Jinan University, Guangzhou, Guangdong 510623, China. ${ }^{5}$ Department of Endocrinology and Metabolism, Guangzhou Women and Children's Medical Center, Guangzhou, Guangdong 510623, China. ${ }^{6}$ Medical Imaging Center, The 1st Affiliated Hospital, Jinan University, Guangzhou, Guangdong 510623, China. ${ }^{7}$ Department of Genetics, Stanford University, Stanford, CA 94305, USA. ${ }^{8}$ Stanley Institute for Cognitive Genomics, Cold Spring Harbor Laboratory, Cold Spring Harbor, NY 11797, USA. ${ }^{9}$ Department of Neurology, The 1st Affiliated Hospital, Jinan University, Guangzhou, Guangdong 510632, China. ${ }^{10}$ Zilkha Neurogenetic Institute, University of Southern California, Los Angeles, CA 90089, USA. ${ }^{11}$ Department of Psychiatry \& Behavioral Sciences, University of Southern California, Los Angeles, CA 90089, USA.

Received: 10 July 2014 Accepted: 20 November 2014

Published online: 03 December 2014

\section{References}

1. Koboldt DC, Steinberg KM, Larson DE, Wilson RK, Mardis ER: The nextgeneration sequencing revolution and its impact on genomics. Cell 2013, 155(1):27-38.

2. Shendure J, Ji H: Next-generation DNA sequencing. Nat Biotechnol 2008, 26(10):1135-1145.

3. Metzker ML: Sequencing technologies - the next generation. Nat Rev Genet 2010, 11(1):31-46.

4. Durbin RM, Abecasis GR, Altshuler DL, Auton A, Brooks LD, Gibbs RA, Hurles $\mathrm{ME}, \mathrm{McV}$ ean GA: A map of human genome variation from populationscale sequencing. Nature 2010, 467(7319):1061-1073.

5. Pool JE, Hellmann I, Jensen JD, Nielsen R: Population genetic inference from genomic sequence variation. Genome Res 2010, 20(3):291-300.

6. Pallen MJ, Loman NJ, Penn CW: High-throughput sequencing and clinical microbiology: progress, opportunities and challenges. Curr Opin Microbiol 2010, 13(5):625-631.

7. Schloss $\mathrm{PD}$, Handelsman J: Metagenomics for studying unculturable microorganisms: cutting the Gordian knot. Genome Biol 2005, 6(8):229.

8. Poland JA, Brown PJ, Sorrells ME, Jannink JL: Development of high-density genetic maps for barley and wheat using a novel two-enzyme genotyping-by-sequencing approach. PLoS One 2012, 7(2):e32253.

9. Goddard ME, Hayes BJ: Mapping genes for complex traits in domestic animals and their use in breeding programmes. Nat Rev Genet 2009, 10(6):381-391.

10. Meaburn E, Schulz R: Next generation sequencing in epigenetics: insights and challenges. Semin Cell Dev Biol 2012, 23(2):192-199.

11. Wang Z, Gerstein M, Snyder M: RNA-Seq: a revolutionary tool for transcriptomics. Nat Rev Genet 2009, 10(1):57-63.

12. Ozsolak F, Milos PM: RNA sequencing: advances, challenges and opportunities. Nat Rev Genet 2011, 12(2):87-98.

13. Bamshad MJ, Ng SB, Bigham AW, Tabor HK, Emond MJ, Nickerson DA, Shendure J: Exome sequencing as a tool for Mendelian disease gene discovery. Nat Rev Genet 2011, 12(11):745-755.

14. Rabbani B, Tekin M, Mahdieh $\mathrm{N}$ : The promise of whole-exome sequencing in medical genetics. J Hum Genet 2014, 59(1):5-15.

15. Ku CS, Naidoo N, Pawitan Y: Revisiting Mendelian disorders through exome sequencing. Hum Genet 2011, 129(4):351-370.

16. Gilissen C, Hoischen A, Brunner HG, Veltman JA: Unlocking Mendelian disease using exome sequencing. Genome Biol 2011, 12(9):228.

17. Yang Y, Muzny DM, Reid JG, Bainbridge MN, Willis A, Ward PA, Braxton A, Beuten J, Xia F, Niu Z, Hardison M, Person R, Bekheirnia MR, Leduc MS, Kirby A, Pham P, Scull J, Wang M, Ding Y, Plon SE, Lupski JR, Beaudet AL, Gibbs RA, Eng CM: Clinical whole-exome sequencing for the diagnosis of mendelian disorders. N Eng/ J Med 2013, 369(16):1502-1511.

18. de Ligt J, Willemsen MH, van Bon BW, Kleefstra T, Yntema HG, Kroes T, Vulto-van Silfhout AT, Koolen DA, de Vries P, Gilissen C, del Rosario M, Hoischen A, Scheffer H, de Vries BB, Brunner HG, Veltman JA, Vissers LE: Diagnostic exome sequencing in persons with severe intellectual disability. N Engl J Med 2012, 367(20):1921-1929. 
19. Rope AF, Wang K, Evjenth R, Xing J, Johnston JJ, Swensen JJ, Johnson WE, Moore B, Huff CD, Bird LM, Carey JC, Opitz JM, Stevens CA, Jiang T, Schank C, Fain HD, Robison R, Dalley B, Chin S, South ST, Pysher TJ, Jorde LB, Hakonarson H, Lillehaug JR, Biesecker LG, Yandell M, Arnesen T, Lyon GJ: Using VAAST to identify an X-linked disorder resulting in lethality in male infants due to $\mathrm{N}$-terminal acetyltransferase deficiency. Am J Hum Genet 2011, 89(1):28-43.

20. Wang K, Kim C, Bradfield J, Guo Y, Toskala E, Otieno FG, Hou C, Thomas K, Cardinale C, Lyon GJ, Golhar R, Hakonarson H: Whole-genome DNA/RNA sequencing identifies truncating mutations in RBCK1 in a novel Mendelian disease with neuromuscular and cardiac involvement. Genome Med 2013, 5(7):67.

21. Nilsson J, Schoser B, Laforet P, Kalev O, Lindberg C, Romero NB, Dávila López M, Akman HO, Wahbi K, Iglseder S, Eggers C, Engel AG, Dimauro S, Oldfors A: Polyglucosan body myopathy caused by defective ubiquitin ligase RBCK1. Ann Neurol 2013, 74(6):914-919.

22. Worthey EA, Mayer AN, Syverson GD, Helbling D, Bonacci BB, Decker B, Serpe JM, Dasu T, Tschannen MR, Veith RL, Basehore MJ, Broeckel U, TomitaMitchell A, Arca MJ, Casper JT, Margolis DA, Bick DP, Hessner MJ, Routes JM, Verbsky JW, Jacob HJ, Dimmock DP: Making a definitive diagnosis: successful clinical application of whole exome sequencing in a child with intractable inflammatory bowel disease. Genet Med 2011, 13(3):255-262.

23. Lyon GJ, O'Rawe J: Human genetics and clinical aspects of neurodevelopmental disorders. In The Genetics of Neurodevelopmental Disorders. Edited by Mitchell K. Hoboken, NJ, USA: Wiley; 2014.

24. Foo JN, Liu JJ, Tan EK: Whole-genome and whole-exome sequencing in neurological diseases. Nat Rev Neurol 2012, 8(9):508-517.

25. Zhao H, Race V, Matthijs G, De Jonghe P, Robberecht W, Lambrechts D, Van Damme P: Exome sequencing reveals HINT1 mutations as a cause of distal hereditary motor neuropathy. Eur J Hum Genet 2014, 22(6):847-850.

26. Beetz C, Pieber TR, Hertel N, Schabhüttl M, Fischer C, Trajanoski S, Graf E, Keiner S, Kurth I, Wieland T, Varga RE, Timmerman V, Reilly MM, Strom TM, Auer-Grumbach M: Exome sequencing identifies a REEP1 mutation involved in distal hereditary motor neuropathy type V. Am J Hum Genet 2012, 91(1):139-145.

27. Klein CJ, Middha S, Duan X, Wu Y, Litchy WJ, Gu W, Dyck PJ, Gavrilova RH, Smith DI, Kocher JP, Dyck PJ: Application of whole exome sequencing in undiagnosed inherited polyneuropathies. J Neurol Neurosurg Psychiatry 2014, 85(11):1265-1272.

28. Stessman HA, Bernier R, Eichler EE: A genotype-first approach to defining the subtypes of a complex disease. Cell 2014, 156(5):872-877.

29. Lyon GJ, Wang K: Identifying disease mutations in genomic medicine settings: current challenges and how to accelerate progress. Genome Med 2012, 4(7):58.

30. Li H: Aligning sequence reads, clone sequences and assembly contigs with BWA-MEM. arXiv 2013, 1303(3997v2):1-3.

31. McKenna A, Hanna M, Banks E, Sivachenko A, Cibulskis K, Kernytsky A, Garimella K, Altshuler D, Gabriel S, Daly M, DePristo MA: The genome analysis toolkit: a MapReduce framework for analyzing next-generation DNA sequencing data. Genome Res 2010, 20(9):1297-1303.

32. Li H, Handsaker B, Wysoker A, Fennell T, Ruan J, Homer N, Marth G, Abecasis $G$, Durbin R: The Sequence Alignment/Map format and SAMtools. Bioinformatics 2009, 25(16):2078-2079.

33. Garrison E, Marth G: Haplotype-based variant detection from short-read sequencing. arXiv Preprint 2012, arXiv:1207-3907. arXiv.

34. Wang K, Li M, Hakonarson H: ANNOVAR: functional annotation of genetic variants from high-throughput sequencing data. Nucleic Acids Res 2010, 38(16):e164.

35. Landrum MJ, Lee JM, Riley GR, Jang W, Rubinstein WS, Church DM, Maglott DR: ClinVar: public archive of relationships among sequence variation and human phenotype. Nucleic Acids Res 2014, 42(Database issue):D980-D985.

36. Untergasser A, Cutcutache I, Koressaar T, Ye J, Faircloth BC, Remm M, Rozen SG: Primer3-new capabilities and interfaces. Nucleic Acids Res 2012, 40(15):e115.

37. Rose PW, Bi C, Bluhm WF, Christie CH, Dimitropoulos D, Dutta S, Green RK, Goodsell DS, Prlic A, Quesada M, Quinn GB, Ramos AG, Westbrook JD, Young J, Zardecki C, Berman HM, Bourne PE: The RCSB protein data bank: new resources for research and education. Nucleic Acids Res 2013, 41(Database issue):D475-D482

38. Ficko-Blean E, Stubbs KA, Nemirovsky O, Vocadlo DJ, Boraston AB: Structural and mechanistic insight into the basis of mucopolysaccharidosis IIIB. Proc Natl Acad Sci U S A 2008, 105(18):6560-6565.
39. Zhang Y: I-TASSER server for protein 3D structure prediction. BMC Bioinform 2008, 9:40.

40. Voznyi $\mathrm{Y}$, Keulemans JL, van Diggelen OP: A fluorimetric enzyme assay for the diagnosis of MPS II (Hunter disease). J Inherit Metab Dis 2001, 24(6):675-680

41. Karpova EA, Voznyi Ya V, Keulemans JL, Hoogeveen AT, Winchester B, Tsvetkova IV, van Diggelen OP: A fluorimetric enzyme assay for the diagnosis of Sanfilippo disease type A (MPS IIIA). J Inherit Metab Dis 1996, 19(3):278-285

42. Marsh J, Fensom AH: 4-Methylumbelliferyl alpha-N-acetylglucosaminidase activity for diagnosis of Sanfilippo B disease. Clin Genet 1985, 27(3):258-262.

43. van Diggelen OP, Zhao H, Kleijer WJ, Janse HC, Poorthuis BJ, van Pelt J, Kamerling JP, Galjaard H: A fluorimetric enzyme assay for the diagnosis of Morquio disease type A (MPS IV A). Clin Chim Acta 1990, 187(2):131-139.

44. Huang YL, Li SY, Zhao XY, Fan LP, Lin WC, Zhou ZH, Cheng J, Liu L: Enzymatic diagnosis and clinical characteristics of 52 children with mucopolysaccharidosis. Zhongguo Dang Dai Er Ke Za Zhi 2012, 14(7):510-514.

45. de Jong JG, Wevers RA, Liebrand-van Sambeek R: Measuring urinary glycosaminoglycans in the presence of protein: an improved screening procedure for mucopolysaccharidoses based on dimethylmethylene blue. Clin Chem 1992, 38(6):803-807.

46. Zhao XY, Huang YL, Li SY, Lin WC, Zhou ZH, Liu L: Quantitative measuring urinary glycosaminoglycan by dimethylmethylene-tris spectrophotometric method. Chin J Child Health Care 2010, 11:885-888.

47. O'Rawe J, Jiang T, Sun G, Wu Y, Wang W, Hu J, Bodily P, Tian L, Hakonarson H, Johnson WE, Wei Z, Wang K, Lyon GJ: Low concordance of multiple variant-calling pipelines: practical implications for exome and genome sequencing. Genome Med 2013, 5(3):28.

48. Liu X, Jian X, Boerwinkle E: dbNSFP: a lightweight database of human nonsynonymous SNPs and their functional predictions. Hum Mutat 2011, 32(8):894-899.

49. Schmidtchen A, Greenberg D, Zhao HG, Li HH, Huang Y, Tieu P, Zhao HZ, Cheng S, Zhao Z, Whitley CB, Di Natale P, Neufeld EF: NAGLU mutations underlying Sanfilippo syndrome type B. Am J Hum Genet 1998, 62(1):64-69.

50. Andria G, Di Natale P, Del Giudice E, Strisciuglio P, Murino P: Sanfilippo B syndrome (MPS III B): mild and severe forms within the same sibship. Clin Genet 1979, 15(6):500-504.

51. Chinen $Y$, Tohma T, Izumikawa $Y$, Uehara H, Ohta T: Sanfilippo type B syndrome: five patients with an R565P homozygous mutation in the alpha-N-acetylglucosaminidase gene from the Okinawa islands in Japan. J Hum Genet 2005, 50(7):357-359.

52. van de Kamp JJ, Niermeijer MF, von Figura K, Giesberts MA: Genetic heterogeneity and clinical variability in the Sanfilippo syndrome (types A, B, and C). Clin Genet 1981, 20(2):152-160

53. Stenson PD, Mort M, Ball EV, Shaw K, Phillips A, Cooper DN: The Human Gene Mutation Database: building a comprehensive mutation repository for clinical and molecular genetics, diagnostic testing and personalized genomic medicine. Hum Genet 2014, 133(1):1-9.

54. Weber B, Guo XH, Kleijer WJ, van de Kamp JJ, Poorthuis BJ, Hopwood J J: Sanfilippo type B syndrome (mucopolysaccharidosis III B): allelic heterogeneity corresponds to the wide spectrum of clinical phenotypes. Eur J Hum Genet 1999, 7(1):34-44.

55. Lee-Chen GJ, Lin SP, Lin SZ, Chuang CK, Hsiao KT, Huang CF, Lien WC: Identification and characterisation of mutations underlying Sanfilippo syndrome type B (mucopolysaccharidosis type IIIB). J Med Genet 2002, 39(2):E3.

56. Nelson J, Crowhurst J, Carey B, Greed L: Incidence of the mucopolysaccharidoses in Western Australia. Am J Med Genet A 2003, 123A(3):310-313.

57. Meikle PJ, Hopwood JJ, Clague AE, Carey WF: Prevalence of lysosomal storage disorders. JAMA 1999, 281(3):249-254.

58. Nelson J: Incidence of the mucopolysaccharidoses in Northern Ireland. Hum Genet 1997, 101(3):355-358.

59. Poorthuis BJ, Wevers RA, Kleijer WJ, Groener JE, de Jong JG, van Weely S, Niezen-Koning KE, van Diggelen OP: The frequency of lysosomal storage diseases in The Netherlands. Hum Genet 1999, 105(1-2):151-156.

doi:10.1186/s12920-014-0066-9

Cite this article as: Shi et al:: "Genotype-first" approaches on a curious case of idiopathic progressive cognitive decline. BMC Medical Genomics 2014 7:66. 